\title{
Antibiorésistance des souches de Salmonella gallinarum isolées en aviculture moderne en zones périurbaines au Mali
}

\author{
Satigui Sidibé ${ }^{*}$ Amadou dit Baba Traoré ${ }^{1}$ Yaya Sidi Koné ${ }^{1}$ \\ Adama Fané ${ }^{1}$ Kadiatou Wele Coulibaly ${ }^{1}$ \\ Adama Broulaye Doumbia ${ }^{1}$ Afou Bamba ${ }^{1}$ Oumar Traoré ${ }^{1}$
}

\begin{abstract}
Mots-clés
Submitted: 4 February 2018

Accepted: 19 August 2019

Published: 15 November 2019

DOI: $10.19182 /$ remvt. 31516
\end{abstract}

Résumé

Volaille, Salmonella gallinarum, résistance aux antibiotiques, Mali

L'objectif de l'étude, menée d'août 2014 à décembre 2015, était de tester la résistance aux antibiotiques de 52 isolats de Salmonella gallinarum obtenus à partir d'élevages avicoles modernes situés en zones périurbaines du district de

Bamako $(n=27)$, et des villes de Ségou $(n=16)$ et Sikasso $(n=9)$. Les taux de résistance moyens obtenus ont été de 98,08 \% à l'érythromycine, 94,23\% à la colistine, 90,38 \% à la streptomycine, 67,31\% à la kanamycine, 65,38\% à la fluméquine, $63,46 \%$ à la doxycycline, 59,61\% à la tétracycline et 21,15\% à la gentamicine. Tous les isolats de salmonelles issus des élevages du district de Bamako se sont avérés résistants à la tétracycline, à la doxycycline et à l'érythromycine. De même, une résistance à l'érythromycine, à la tétracycline, à la colistine et à la streptomycine a été mise en évidence pour tous les isolats issus des élevages du site de Sikasso. Les résultats ont montré un développement de la résistance de la plupart des souches de salmonelles isolées à la majorité des antibiotiques usuels et dans une moindre mesure à la gentamicine.

- Comment citer ce article: Sidibé S., Traoré A.B., Koné Y.S., Fané A., Coulibaly K.W., Doumbia A.B . Bamba A., Traoré O., 2019. Antibiotic resistance of isolated Salmonella gallinarum strains in modern poultry farming in suburban areas in Mali. Rev. Elev. Med. Vet. Pays Trop., 72 (4): 167-171, doi: 10.19182/ remvt.31516

\section{INTRODUCTION}

Le cheptel aviaire au Mali compte près de 38590000 têtes, toutes espèces confondues (DNPIA, 2016). Le secteur moderne représente plus de $10 \%$ de cet effectif et est constitué d'exploitations comportant jusqu'à 30000 têtes (DNPIA, 2016). L'aviculture moderne est la principale source d'approvisionnement des populations en œufs et poulets de chair. A la différence des fermes avicoles traditionnelles villageoises, ces exploitations hébergent des effectifs plus importants par bandes, composés principalement de races exotiques de poules pondeuses dont la Leghorn, la Lhomann Rouge, la Rhode Island, ou de poulets de chair de races Cobb 500 ou Ross élevés sur des espaces

1. Laboratoire central vétérinaire, BP 2295, Bamako, Mali.

* Auteur pour la correspondance

Tél. : +22320243344 / 20246653

Email : sidibsatigui@gmail.com

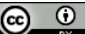

https://creativecommons.org/licenses/by/4.0/ assez réduits (7-8 volailles $\left./ \mathrm{m}^{2}\right)$; elles bénéficient de meilleures conditions d'habitat, d'hygiène, d'alimentation et de suivi sanitaire.

Les poussins sont en général importés, même si des embryons d'unités industrielles de production locale de poussins d'un jour commencent à émerger. Bien que les fournisseurs de poussins mettent à la disposition des aviculteurs des plans de prophylaxie, les volailles continuent d'être lourdement impactées par les pathologies, notamment les salmonelloses (Arbelot., 1997 ; Arbelot et al., 1997) qui constituent une préoccupation dans les élevages avicoles africains (Allaoui et al., 2017 ; Elared et al., 2001 ; Arbelot, 1997 ; Arbelot et al., 1997 ; Kounta, 1992 ; Kounta, 1993). Ainsi, des salmonelles ont été isolées en culture dans plusieurs pays d'Afrique subsaharienne aussi bien à partir d'élevage que sur des abats ou sur les carcasses de volailles (Combari, 2014 ; Toko, 2010 ; Coulibaly et al., 2005).

En Afrique de l'Ouest, les salmonelloses causent d'importantes pertes économiques lors de mortalités pouvant atteindre $90 \%$. En outre, les humains peuvent également être contaminés à travers la consommation de produits issus de volailles (Kimura et al., 2004 ; Sannat et al., 2017 ; Cardinale et al., 2005). Au Mali, les salmonelloses à 
S. pullorum/gallinarum font partie des pathologies dominantes en aviculture moderne avec des taux de prévalences respectivement de 20,09\% et de 19,53\% pour les infections d'organes et d'œufs (Sidibé et al., 2013). La lutte contre les salmonelloses aviaires en Afrique est surtout basée sur l'utilisation abusive de produits antimicrobiens, en l'occurrence les antibiotiques. Pour une production alimentaire sans salmonelles, Awad et Ghareeb (2014) recommandent, entre autres, l'application de la vaccination et de mesures de biosécurité. Au Mali, notamment dans les petites exploitations avicoles, les mesures d'hygiène et de biosécurité pour minimiser le risque d'introduction et de diffusion d'agents pathogènes ne sont pas correctement appliquées. De même, la vaccination contre les salmonelloses n'est pas systématique en raison de son coût.

Dans ce contexte, différents complexes médicamenteux (Neotreat, Panteryl, Tetracolivit, Alfacéryl et Gentadox) renfermant un ou plusieurs antibiotiques, comme l'érythromycine, la colistine, la streptomycine, la doxycycline, la tétracycline, la gentamicine, sont fréquemment utilisés pour prévenir ou traiter les salmonelloses en aviculture moderne. Ils sont également utilisés chez la volaille comme promoteurs de croissance. Suite à la persistance de cas de salmonelloses dans les exploitations avicoles modernes, plusieurs cas d'échecs thérapeutiques ont été observés après l'usage de ces médicaments. Par ailleurs, le profil de résistance aux antibiotiques des souches de salmonelles isolées demeure mal connu.

L'usage incontrôlé d'antibiotiques peut conduire à une sélection de bactéries pathogènes résistantes (Sanders et al., 2012). De même, l'augmentation de l'incidence des souches de Salmonella résistantes aux antibiotiques dans les élevages semi-intensifs a été évoquée par Casin et al. (1996). L'antibiorésistance est devenue un sérieux problème de santé publique. L'objectif de la présente étude était de déterminer le profil de résistance à huit antibiotiques usuels (gentamicine, fluméquine, tétracycline, doxycycline, colistine, streptomycine, érythromycine et kanamycine) de souches de $S$. gallinarum isolées dans des élevages avicoles modernes.

\section{- MATERIEL ET METHODES}

L'étude a été réalisée d'août 2014 à décembre 2015 en deux phases : le suivi clinique épidémiologique et la collecte de prélèvements, puis les examens de laboratoire.

\section{Choix des sites et des élevages}

Cette étude prospective a été réalisée dans les zones périurbaines du district de Bamako et des villes de Ségou et Sikasso (figure 1). Le développement et l'importance de l'aviculture moderne dans ces sites ont justifié ces choix. En zone périurbaine de Bamako, 96 élevages avicoles ont été visités sur six axes : Bamako-Koulikoro, Bamako-Siby, Bamako-Kangaba, Bamako-Ségou, Bamako-Kati et Bamako-Bougouni. A Ségou, 43 élevages ont été choisis sur trois axes : Ségou-Markala, Ségou-Cinzana, Ségou-Bamako. A Sikasso, 43 fermes avicoles ont été ciblées sur quatre axes : Sikasso-Koutiala, Sikasso-Nièna, Sikasso - Bobo Dioulasso, Sikasso-Missiricoro. Les élevages ont été choisis en fonction de l'importance des effectifs de volailles qui variaient entre 1000 et 30000 têtes, du type de spéculation (poules pondeuses), de l'adhésion des aviculteurs à l'esprit de l'étude, de l'accessibilité des élevages et de la fréquence de cas suspects de salmonelloses.

\section{Collecte des échantillons}

Au cours de visites bimensuelles, des écouvillonnages cloacaux ont été réalisés sur les poules pondeuses présentant des signes cliniques suspects de salmonelloses (dépression, anorexie, diarrhées

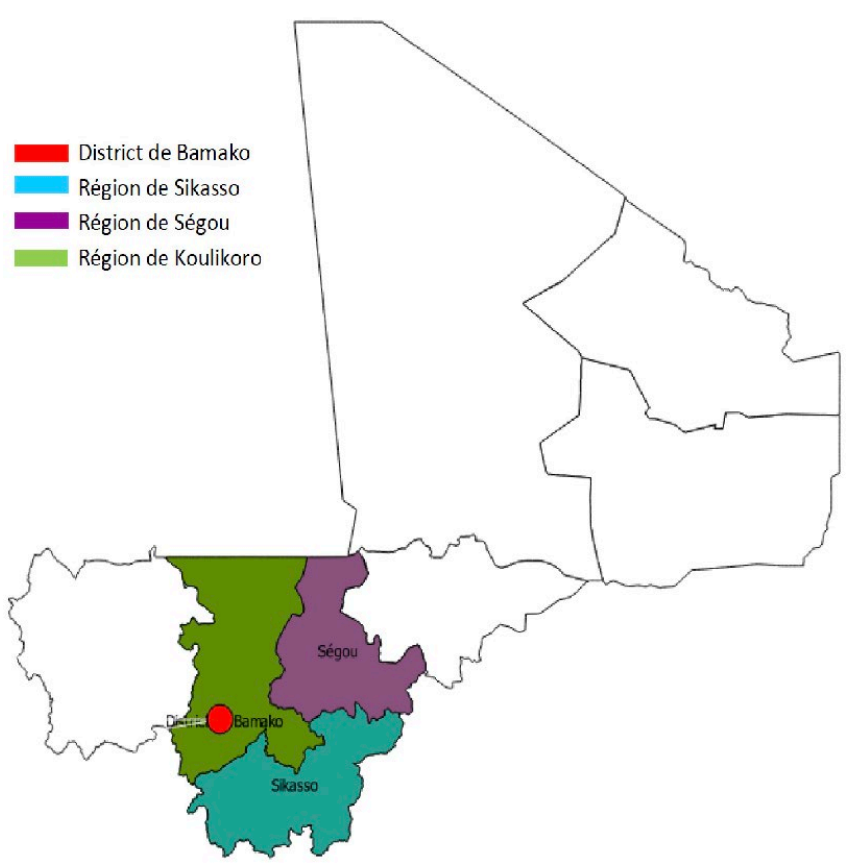

Figure 1 : localisation des trois sites de l'étude au Mali.

blanchâtres, déshydratation, anémie et cyanose sur la crête et les barbillons). Des prélèvements d'organes (foie, rate, intestins, grappes ovariennes, poumons) ont été collectés en cas de présence de signes lésionnels spécifiques de la maladie (décoloration du foie, ovarite, salpingite, splénomégalie, entérite, myopéricardite) à l'autopsie de sujets malades ou récemment morts (moins de quatre heures). Les prélèvements ont été identifiés en notant les informations relatives à l'élevage, aux signes cliniques et lésionnels, et à la nature du prélèvement. Ils ont ensuite été conservés sous glace et acheminés au Laboratoire central vétérinaire pour les examens. Au total, 526 prélèvements, dont 515 écouvillons cloacaux et 11 prélèvements d'organes, ont été collectés dans 182 élevages (tableau I).

\section{Méthodes d'analyse de laboratoire}

\section{Recherche des salmonelles}

L'analyse des échantillons soumis au laboratoire a été effectuée en trois étapes. D'abord, chaque écouvillon a été trempé dans de l'eau peptonée, puis incubé à $37^{\circ} \mathrm{C}$ pendant $24 \mathrm{~h}$. Les organes ont été au préalable broyés, dilués à $10 \%(\mathrm{P} / \mathrm{V})$ dans de l'eau peptonée $(1 \mathrm{ml} / \mathrm{g}$ de broyat pesé) puis incubés pendant $24 \mathrm{~h}$. Ensuite, le bouillon au tétrathionate a été ensemencé avec un millilitre de culture de préenrichissement sur eau peptonée, puis incubé à $37^{\circ} \mathrm{C}$ pendant $24 \mathrm{~h}$. Enfin, à partir des cultures obtenues sur les milieux d'enrichissement, les milieux sélectifs, comme les géloses Mac Conkey et SalmonellaShigella, ont été ensemencés, puis incubés à $37^{\circ} \mathrm{C}$ pendant $24 \mathrm{~h}$. Les

\section{Tableau I}

Nombre d'échantillons collectés chez des poules pondeuses en zones périurbaines au Mali

\begin{tabular}{lcccc} 
Echantillon & Bamako & Ségou & Sikasso & Total \\
\hline Ecouvillon cloacal & 273 & 98 & 144 & 515 \\
Prélèvement d'organes & 04 & 01 & 06 & 11 \\
Total & 277 & 99 & 150 & 526
\end{tabular}


colonies caractéristiques de Salmonella, paraissant incolores avec un centre noir sur géloses Salmonella-Shigella et Mac Conkey, ont été observées. Les cultures pures obtenues sur gélose tryptone soja ont été utilisées pour la réalisation de tests biochimiques classiques ou de systèmes API (galeries biochimiques miniaturisées).

\section{Technique de l'antibiogramme}

Le test de sensibilité aux antibiotiques a été effectué selon la méthode de diffusion en milieu gélosé de Kirby-Bauer et les recommandations du Comité de l'antibiogramme de la société française de microbiologie (CA-SFM). Le profil de résistance à huit antibiotiques présents dans des complexes médicamenteux couramment utilisés en aviculture moderne a été déterminé : la gentamicine $(10 \mu \mathrm{g})$, la fluméquine $(30 \mu \mathrm{g})$, la kanamycine $(30 \mu \mathrm{g})$, la tétracycline $(30 \mu \mathrm{g})$, la doxycycline $(30 \mu \mathrm{g})$, la colistine $(10 \mu \mathrm{g})$, la streptomycine $(10 \mu \mathrm{g})$ et l'érythromycine $(15 \mu \mathrm{g})$. Pour ce faire, chaque souche a été repiquée sur gélose tryptone soja puis incubée à $37^{\circ} \mathrm{C}$ pendant $24 \mathrm{~h}$ afin d'obtenir des cultures pures. A partir de colonies de la culture de $24 \mathrm{~h}$, une suspension turbide a été réalisée dans deux millilitres d'eau physiologique $(\mathrm{NaCl} 85 \%)$ à l'échelle 0,5 de Mac Farland. L'inoculum a été obtenu en émulsionnant $100 \mu \mathrm{l}$ de cette suspension dans $10 \mathrm{ml}$ d'eau physiologique. La gélose Mueller Hinton en boîte de Petri a été ensemencée par strie à l'aide d'écouvillons imbibés de suspension bactérienne. Les disques des huit antibiotiques ont été placés à l'aide d'un distributeur de disques. Les boîtes contenant les disques ont été laissées à la température ambiante sous hotte pendant $30 \mathrm{~min}$ puis incubées pendant $24 \mathrm{~h}$ à $37^{\circ} \mathrm{C}$. Les résultats ont été interprétés comme sensibles, intermédiaires ou résistants en fonction du diamètre d'inhibition sur gélose Mueller Hinton selon les recommandations du CA-SFM et la méthode de Quinn et al. (1994) (tableau II). Cependant, seuls les résultats résistants et sensibles ont été considérés lors de la synthèse des tests d'antibiogramme. La doxycycline et la fluméquine ne figurent pas sur la fiche de lecture proposée par Quinn et al. (1994), expliquant leur absence du tableau II.

\section{Tableau II}

Interprétation des résultats de tests de résistance de souches de Salmonella gallinarum à six antibiotiques selon Quinn et al. (1994)

\begin{tabular}{lrrc} 
Antibiotique & Resistant $\leq$ & Intermédiaire & Sensible $\geq$ \\
\hline Gentamicine $(10 \mu \mathrm{g})$ & 12 & $13-14$ & 15 \\
Kanamycine $(30 \mu \mathrm{g})$ & 13 & $14-17$ & 18 \\
Tétracycline $(30 \mu \mathrm{g})$ & 14 & $15-18$ & 19 \\
Colistine $(10 \mu \mathrm{g})$ & 8 & $9-10$ & 11 \\
Streptomycine $(10 \mu \mathrm{g})$ & 11 & $12-14$ & 15 \\
Erythromycine $(15 \mu \mathrm{g})$ & 13 & $14-22$ & 23
\end{tabular}

\section{RESULTATS}

La mise en culture des 526 prélèvements a permis d'établir un taux de prévalence bactériologique global de 9,9\% (52 cas positifs) pour S. gallinarum. Par ailleurs, 27 échantillons provenant de 96 élevages de la zone périurbaine du district de Bamako, 15 échantillons issus de 43 élevages de la zone de Ségou et 12 échantillons issus de 43 élevages de la zone de Sikasso ont été positifs à la culture des salmonelles. Les résultats relatifs au profil de résistance de $S$. gallinarum aux huit antibiotiques sont présentés dans le tableau III. En moyenne, pour l'ensemble des trois sites, les taux de résistance ont été de 98,1\%

\section{Tableau III}

Profil de résistance des souches de Salmonella gallinarum isolées chez des poules pondeuses en zones périurbaines au Mali

\begin{tabular}{lcccc} 
Antibiotique & $\begin{array}{c}\text { Bamako } \\
\mathbf{n = 2 7} \\
(\mathbf{\%})\end{array}$ & $\begin{array}{c}\text { Ségou } \\
\mathbf{n = 1 6} \\
\mathbf{( \% )}\end{array}$ & $\begin{array}{c}\text { Sikasso } \\
\mathbf{n = 9} \\
\mathbf{( \% )}\end{array}$ & $\begin{array}{c}\text { Total } \\
\mathbf{n = 5 2} \\
\mathbf{( \% )}\end{array}$ \\
\hline $\begin{array}{l}\text { Gentamicine } \\
(10 \mu \mathrm{g})\end{array}$ & $6(22,22)$ & $3(18,70)$ & $2(22,22)$ & $11(21,15)$ \\
$\begin{array}{l}\text { Fluméquine } \\
(30 \mu \mathrm{g})\end{array}$ & $16(59,25)$ & $11(68,70)$ & $7(77,77)$ & $34(65,38)$ \\
$\begin{array}{l}\text { Kanamycine } \\
(30 \mu \mathrm{g})\end{array}$ & $19(70,37)$ & $9(56,25)$ & $7(77,77)$ & $35(67,31)$ \\
$\begin{array}{l}\text { Tétracycline } \\
(30 \mu \mathrm{g})\end{array}$ & $22(81,48)$ & 0 & $9(100)$ & $31(59,61)$ \\
$\begin{array}{l}\text { Doxycycline } \\
(30 \mu \mathrm{g})\end{array}$ & $25(92,59)$ & 0 & $8(88,88)$ & $33(63,46)$ \\
$\begin{array}{l}\text { Colistine } \\
(10 \mu \mathrm{g})\end{array}$ & $25(92,59)$ & $15(93,75)$ & $9(100)$ & $49(94,23)$ \\
$\begin{array}{l}\text { Streptomycine } \\
(10 \mu \mathrm{g})\end{array}$ & $25(92,59)$ & $13(81,27)$ & $9(100)$ & $47(90,38)$ \\
$\begin{array}{l}\text { Erythromycine } \\
(15 \mu \mathrm{g})\end{array}$ & $26(96,29)$ & 0 & $9(100)$ & $51(98,08)$
\end{tabular}

à l'érythromycine, 94,2 \% à la colistine, 90,4\% à la streptomycine, $67,3 \%$ à la kanamycine, 65,4 \% à la fluméquine, 63,5\% à la doxycycline, 59,6\% à la tétracycline et 21,2\% à la gentamicine. Pour les 27 isolats de Bamako, le taux de résistance à l'érythromycine a été le plus élevé $(96,3 \%)$, suivi de ceux à la doxycycline, à la colistine et à la streptomycine (92,6\% chacun). Sur 16 isolats obtenus à Ségou, le taux de résistance à la gentamicine a été de $18,7 \%$ et celui à la colistine de 93,7\% a été le plus élevé. Tous les isolats de ce site se sont avérés sensibles à la tétracycline, à la doxycycline et à l'érythromycine. Enfin à Sikasso, sur neuf isolats de $S$. gallinarum mis en évidence, le taux de résistance à la doxycycline $(88,9 \%)$ a été le plus élevé.

\section{- DISCUSSION}

L'analyse du profil de résistance aux antibiotiques usuels sur des isolats de $S$. gallinarum a montré que les taux de résistance les plus élevés étaient avec l'érythromycine (98,1\%), puis la colistine $(94,2 \%)$ et la streptomycine $(90,4 \%)$. Celui pour l'érythromycine était cependant inférieur à celui observé par Combari au Sénégal (2014) dans les fermes avicoles de la zone périurbaine de Dakar, qui rapporte que $100 \%$ des souches de salmonelles étaient résistantes à cet antibiotique. Cette similarité pourrait s'expliquer par l'utilisation abusive des promoteurs de croissance contenant en majorité de la colistine et de la streptomycine, et à la résistance naturelle des bactéries à Gram négatif à l'érythromycine. Le pourcentage de résistance le plus faible a été observé pour la gentamicine $(21,2 \%)$. Il était comparable à celui de 8,8 \% obtenu par Elared et al. (2001) au Maroc. Cet antibiotique est très peu utilisé en aviculture à cause de son coût élevé. Le taux de résistance aux tétracyclines (59,6\%) était comparable à celui obtenu au Sénégal par Combari (2014) et inférieur à celui de Toko (2010) en Côte d'Ivoire (100\%). Cette différence pourrait s'expliquer par le niveau d'utilisation des antibiotiques qui varie d'un pays à l'autre.

En France, il a été établi que les résistances aux tétracyclines sont les plus élevées (ANSES, 2010). Ces dernières sont d'usage courant 
en thérapeutique vétérinaire et appartiennent à la famille d'antibiotiques la plus utilisée chez les humains. La mise en évidence d'isolats de $S$. gallinarum résistants à la fluméquine $(62,6 \%$ ) (classe des fluoroquinolones) est très inquiétante car cette famille d'antibiotiques est utilisée en derniers recours lors du traitement des salmonelloses humaines graves (Weill, 2008). Ce taux était supérieur à celui de 25,5\% obtenu par Combari (2014) au Sénégal.

Le développement de la résistance antimicrobienne à l'échelle mondiale a conduit l'Organisation mondiale de la santé (OMS, 2016), l'Organisation mondiale de la santé animale (OIE), et l'Organisation des Nations unies pour l'alimentation et l'agriculture (ONU-FAO) à adopter une approche concertée en vue d'élaborer un plan mondial pour combattre la résistance aux antimicrobiens. Au Mali, l'élaboration d'un «Plan d'action national de lutte contre la résistance aux antimicrobiens » est en cours de finalisation. Ce plan prévoit la mise en place d'un cadre législatif pour une meilleure lutte contre la résistance antimicrobienne dans le pays. Ainsi, la mise en œuvre des activités clés du plan (information, éducation, sensibilisation des acteurs sur l'ampleur et les mesures de contrôle du phénomène, réglementation rigoureuse de la vente et de l'usage des antibiotiques) contribuerait à réduire le développement de la résistance antimicrobienne. Des campagnes de sensibilisation et de formation des utilisateurs doivent transmettre des messages visant à éviter l'usage d'antibiotiques comme facteurs de croissance, de ne les utiliser qu'en cas de salmonellose confirmée suivie d'un antibiogramme, et de procéder à l'usage rationnel et responsable des antibiotiques.

\section{REFERENCES}

Agence nationale de sécurité sanitaire de l'alimentation, de l'environnement et du travail, 2013. Résapath - Réseau d'épidémiosurveillance de I'antibiorésistance des bactéries pathogènes animales. Bilan 2012. ANSES, Maisons-Alfort, France, $164 \mathrm{p}$

Allaoui A. El, Filali F.R., Ameur N., Bouchrif B., 2017. Contamination des élevages de dinde de chair par Salmonella spp. au Maroc : prévalence, antibiorésistance et facteurs de risque associés. Rev. Sci. Tech. Off. Epizoot. 36 (3) : 935-946, doi : 10.20506/rst.36.3.2726

Arbelot B., 1997. Rapport de fin de contrat. Laboratoire de pathologie aviaire ISRA LNERV, Prodec 5B. Cirad-EMVT, Montpellier, France

Arbelot B., Dayon J.F., Mamis D., Gueye J.C., Tall F., Samb H., 1997. Seroprevalence survey of dominant avian diseases in Senegal: mycoplasmoses, fowl typhoid and pullorum disease, Newcastle, infectious bursal and infectious bronchitis diseases. Rev. Elev. Med. Vet. Pays Trop., 50 (3): 197 203, doi: 10.19182/remvt.9569

Awad W.A., Ghareeb K., 2014. Some aspects of control of Salmonella infection in poultry for minimizing contamination in food chain. Salmonella control in poultry industry. World Poult. Sci. J., 70 (3): 519-530, doi $10.1017 /$ S0043933914000579

Cardinale E., Perrier Gros-Claude J.D., Rivoal K., Rose V., Tall F., Mead G.C., Salvat G., 2005. Epidemiological analysis of Salmonella enterica ssp. enterica serovars Hadar, Brancaster and enteritidis from humans and broile chickens in Senegal using pulsed-field gel electrophoresis and antibiotic susceptibility. J. Appl. Microbiol., 99: 968-977, doi: 10.1111/j.13652672.2005.02618.x

Casin I., Brisabois A., Berger N., Breuil J., Collatz E., 1996. Phénotypes et génotypes de résistance de 182 souches de Salmonella sérotype Typhimurium résistantes à I'ampicilline d'origine humaine et animale. Med. Mal. Infect., 26 : 426-430, doi : 10.1016/S0399-077X(96)80187-9

Combari A., 2014. Niveau de contamination par les salmonelles antibiorésistantes des élevages de poulet de chair en zone péri-urbaine de Dakar. Thèse Doct. Méd. Vét., Ecole inter-Etat des sciences et médecines vétérinaires, Dakar, Sénégal, 64 p.

Coulibaly E.K., Bakayogo S., Karou T.G., Coulibaly K.J., Goualie G.B., Dosso M., Diopoh K.J., 2005. Sérotypage et antibiorésistance des souches de Salmonella isolées dans les foies de poulet vendus sur les marchés de Yopougon (Abidjan, Côte d'Ivoire) en 2005. Rev. Afr. Santé Prod. Anim., 8 (s)

\section{CONCLUSION}

La majorité des souches de $S$. gallinarum isolées dans les trois sites de l'étude se sont révélées résistantes à la plupart des antibiotiques usuels. Les techniciens avicoles doivent privilégier la réalisation d'antibiogrammes avant d'entreprendre des traitements. Dans le cadre de la thérapeutique des salmonelloses, l'utilisation de produits à base de gentamicine doit être privilégiée. Des études complémentaires doivent être réalisées en élargissant le test d'antibiogramme à un nombre plus important de sites, en vue d'obtenir des résultats plus complets sur le profil de résistance des salmonelles en aviculture moderne au Mali.

\section{Remerciements}

Les auteurs adressent leurs sincères remerciements au Gouvernement du Mali pour son appui financier à travers le fonds « Etudes et Recherches », et M. D.D. Dakouo, technicien de laboratoire, pour son appui à la réalisation des examens de laboratoire.

\section{Déclaration des contributions des auteurs}

SS a coordonné les travaux de conception, de planification de l'étude, et la mise en œuvre des analyses de laboratoire ; YSK et AF ont participé à la conception, planification et réalisation de l'étude ; ABT et ABD ont participé à la collecte des prélèvements sur le terrain, et à la rédaction de l'article; $\mathrm{ABT}, \mathrm{ABD}$ et KWC ont participé à la mise en œuvre des examens de laboratoire ; AB et OT ont participé aux analyses de laboratoire.
Direction nationale des productions et industries animales, 2016. Rapport annuel d'activités. DNPIA, Bamako, Mali, 137 p.

Elared O., Amara A., Faid M., Alaoui M.A., Tahri El H., 2001. Antibiorésistance des souches de Salmonella enteritidis isolées dans la zone Rabat-Casablanca à partir de l'œuf de consommation, de l'aliment et des fientes de poules pondeuses. Actes Inst. Agron. Vet (Maroc)., 21 (3) : 147-150

Kimura A.C., Reddy V., Marcus R., Cieslak P.R., Moehle-Boetani J.C., Kassenborg H.D., Segler S.D., et al., 2004. Chicken consumption is a newly identified risk factor for sporadic Salmonella enterica serotype Enteridis infections in the United States: a case-control study in Foodnet sites. Clin. Infect. Dis., 38 (Suppl. 3): 244-252, doi: 10.1086/381576

Kounta A.O.S., 1992. Note technique sur le développement de l'aviculture au Mali. Tropicultura, 10 : 103-105

Kounta A.O.S., 1993. Vers un développement de I'aviculture villageoise au Mali. Afric. Agric. (223) : 56

OMS, 2016. Plan d'action mondial pour combattre la résistance aux antimicrobiens. OMS, Genève, Suisse, $32 \mathrm{p}$.

Quinn P.J., Carter M.E., Markey B., Carter G.R., 1994. Clinical veterinary microbiology. Wolfe, London, UK

Sanders P., Granier S.A., Blanc-Gonnet A., Santolini J., 2012. Les plans de surveillance de l'antibiorésistance en santé animale : le contexte européen et les évolutions récentes. Bull. Epidémiol. Santé Anim. Aliment. (53 Spécial Antibiot. Antibiorésistances) : 25-29

Sannat C., Patyal A., Rawat N., Ghosh R.C., Jolhe D.K., Shende R.K., Hirpurkar S.D., et al., 2017. Characterization of Salmonella Gallinarum from an outbreak in Raigarh, Chhattisgarh. Vet. World, 10 (2): 144-148, doi: 10.14202/vetworld.2017.144-148

Sidibé S.S., Coulibaly M.K.., Sylla M., Traoré B., Kéita S., Doumbia M.R., Diallo M., et al., 2013. Pathologies dominantes identifiées en aviculture semi-industrielle au Mali (zones périurbaines du District de Bamako et des villes de Sikasso et Ségou). Bull Anim. Health Prod. Afr., 61 (3) : 427-433

Toko A., 2010. Evaluation du niveau de résistance de salmonelles d'origine aviaire vis-à-vis de la tétracycline et du sulfaméthoxazole. Thèse Doct. Méd. Vét., Ecole inter-Etat des sciences et médecines vétérinaires, Dakar, Sénégal, $84 \mathrm{p}$.

Weill F.X., 2008. Salmonelles non-typhiques d'origine animale et résistance aux antibiotiques. Bull. Acad. Vét. France, 61 (3), doi : $10.4267 / 2042 / 47946$ 


\section{Summary}

Sidibé S., Traoré A.B., Koné Y.S., Fané A., Coulibaly K.W., Doumbia A.B., Bamba A., Traoré O. Antibiotic resistance of isolated Salmonella gallinarum strains in modern poultry farming in suburban areas in Mali

The objective of the study, conducted from August 2014 to December 2015, was to test the antibiotic resistance of 52 Salmonella gallinarum isolates obtained from modern poultry farms located in suburban areas of Bamako District $(n=27)$, and of Segou ( $\mathrm{n}=16)$ and Sikasso $(\mathrm{n}=9)$ cities. The average resistance rates obtained were $98.08 \%$ to erythromycin, $94.23 \%$ to colistin, $90.38 \%$ to streptomycin, $67.31 \%$ to kanamycin, $65.38 \%$ to flumequine, $63.46 \%$ to doxycycline, $59.61 \%$ to tetracycline and $21.15 \%$ to gentamicin. All Salmonella isolates from Bamako District farms were resistant to tetracycline, doxycycline and erythromycin. Similarly, all isolates from Sikasso area farms showed resistance to erythromycin, tetracycline, colistine and streptomycin. The results showed a development of resistance of most isolated salmonella strains to the majority of common antibiotics, and to a lesser extent to gentamicin.

Keywords: poultry, Salmonella gallinarum, resistance to antibiotics, Mali

\section{Resumen}

Sidibé S., Traoré A.B., Koné Y.S., Fané A., Coulibaly K.W., Doumbia A.B., Bamba A., Traoré O. Resistencia a los antibióticos de cepas de Salmonella gallinarum aisladas en avicultura moderna en zonas periurbanas en Mali

El objetivo de este estudio, Ilevado a cabo entre agosto 2014 y diciembre 2015, fue el de examinar la resistencia a antibióticos en 52 aislamientos de Salmonella gallinarum, obtenidos a partir de granjas avícolas modernas, situadas en zonas periurbanas del distrito de Bamako $(n=27)$, y de las ciudades de Segou $(n=16)$ y Sikasso $(n=9)$. Las tasas de resistencia promedio obtenidas fueron de $98,08 \%$ a la eritromicina, 94,23\% a la colistina, 90,38 a la estreptomicina, 67,31 a la kanamicina, $65,38 \%$ a la flumequina, $63,46 \%$ a la doxiciclina, $59,61 \%$ a la tetraciclina y $21,15 \%$ a la gentamicina. Todos los aislamientos de salmonelas provenientes de granjas del distrito de Bamako fueron resistentes a la tetraciclina, a la doxiciclina y a la eritromicina. También se demostró una resistencia a la eritromicina, a la tetraciclina, a la colistina y a la estreptomicina en todos los aislamientos provenientes de Sikasso. Los resultados mostraron un desarrollo de la resistencia en la mayoría de las cepas de salmonelas aisladas a la mayoría de los antibióticos usuales y en menor medida a la gentamicina.

Palabras clave: aves de corral, Salmonella gallinarum, resistencia a los antibióticos, Malí 
